\title{
Validation of Diffusion Tensor Magnetic Resonance Axonal Fiber Imaging with Registered Manganese-Enhanced Optic Tracts
}

\author{
Ching-Po Lin,* Wen-Yih Isaac Tseng, $\dagger^{, 1}$ Hui-Cheng Cheng, $\ddagger$ and J yh-Horng Chen*
}

*Interdisciplinary MRI/MRS Lab, Department of Electrical Engineering and †Center for Optoelectronic Biomedicine, National Taiwan University, Taipei, Taiwan; and ‡Department of Medical Education and Research, Taipei Veterans General Hospital, Taipei, Taiwan

Received J anuary 23, 2001

Noninvasive mapping of white matter tracts using diffusion tensor magnetic resonance imaging (DTMRI) is potentially useful in revealing anatomical connectivity in the human brain. However, a gold standard for validating DTMRI in defining axonal fiber orientation is still lacking. This study presents the first validation of the principal eigenvector of the diffusion tensor in defining axonal fiber orientation by superimposing DTMR I with manganese-enhanced MRI of optic tracts. A rat model was developed in which optic tracts were enhanced by manganese ions. Manganese ion $\left(\mathrm{Mn}^{2+}\right)$ is a potent $\mathrm{T} 1$-shortening agent and can be uptaken and transported actively along the axon. Based on this property, we obtained enhanced optic tracts with a T1-weighted spin-echo sequence $10 \mathrm{~h}$ after intravitreal injection of $\mathrm{Mn}^{2+}$. The images were compared with DTMRI acquired with exact spatial registration. Deviation angles between tangential vectors of the enhanced tracts and the principal eigenvectors of the diffusion tensor were then computed pixel by pixel. We found that under signal-to-noise (SNR) of 30, the variance of deviation angles was $\left(13.27^{\circ}\right)^{2}$. In addition, the dependence of this variance on SNR obeys stochastic behavior if SNR is greater than 10. Based on this relation, we estimated that an rms deviation of less than $10^{\circ}$ could be achieved with DTMRI when SNR is $\mathbf{4 0}$ or greater. In conclusion, our method bypasses technical difficulties in conventional histological approach and provides an in vivo gold standard for validating DTMRI in mapping white matter tracts. ๑ 2001 Academic Press

Key Words: diffusion tensor; validation; $\mathrm{Mn}^{2+}$; MRI.

${ }^{1}$ To whom correspondence should be addressed at National Taiwan University Medical College, Center for Optoelectronic Biomedicine, 1 J en-Ai Road, Sec. 1, Taipe, Taiwan, ROC. Fax: 886-2-23926922. E-mail: wytseng@ha.mc.ntu.edu.tw.

\section{INTRODUCTION}

Study of neural connectivity is essential for understanding development, function, and plasticity following experience or adverse insults in the brain (Rye, 1999; Friston et al., 1997; Werring et al., 1998). Conventionally, tracers are infused into a specific brain region and anterograde or retrograde transport of tracers along specific fascicles hel ps define the neural connectivity of a nucleus with its target or origin. The invasiveness of this approach, however, restricts its application to animals or post mortem human brains (Dejerine, 1895; Turner et al., 1980; Y oung et al., 1995; Pautler et al., 1998). Recent advance in diffusion tensor magnetic resonance imaging (DTMRI) allows probing direction-dependent diffusivity of water molecules in the white matter and is thus potentially useful in determining neural fiber tracts noninvasively (Douek et al., 1991; Basser et al., 1994; Beaulieu et al., 1994; Makris et al., 1997). This application assumes parallel relationships between the direction of highest diffusion, namely, the principal eigenvector of the diffusion tensor, and the direction of fiber fascicles traversing the imaged voxel. Algorithms of three-dimensional (3-D) tractography reconstructed from DTMRI are under active development, and initial results of mapping connectivity in vivo have been reported (Basser et al., 1998, 2000; Conturo et al., 1999; J ones et al., 1999; Mori et al., 1999; Xue et al., 1999).

In vivo protocols for DTMRI acquisition suffer from some physical constraints and artifacts that compromise the accuracy of the principal eigenvector of the diffusion tensor. The principal eigenvector would not be able to reflect the underlying neural fiber direction if the spatial resolution is insufficient to resolve the partial volume effect, usually in the gray-white matter junction or in areas of high curvature fiber bundles or tract crossing (Pierpaoli et al., 1996; Tuch et al., 1999; Wiegell et al., 2000). These voxels create ambiguous directions in DTMRI and limit the extent of tract reconstruction. Artifacts 

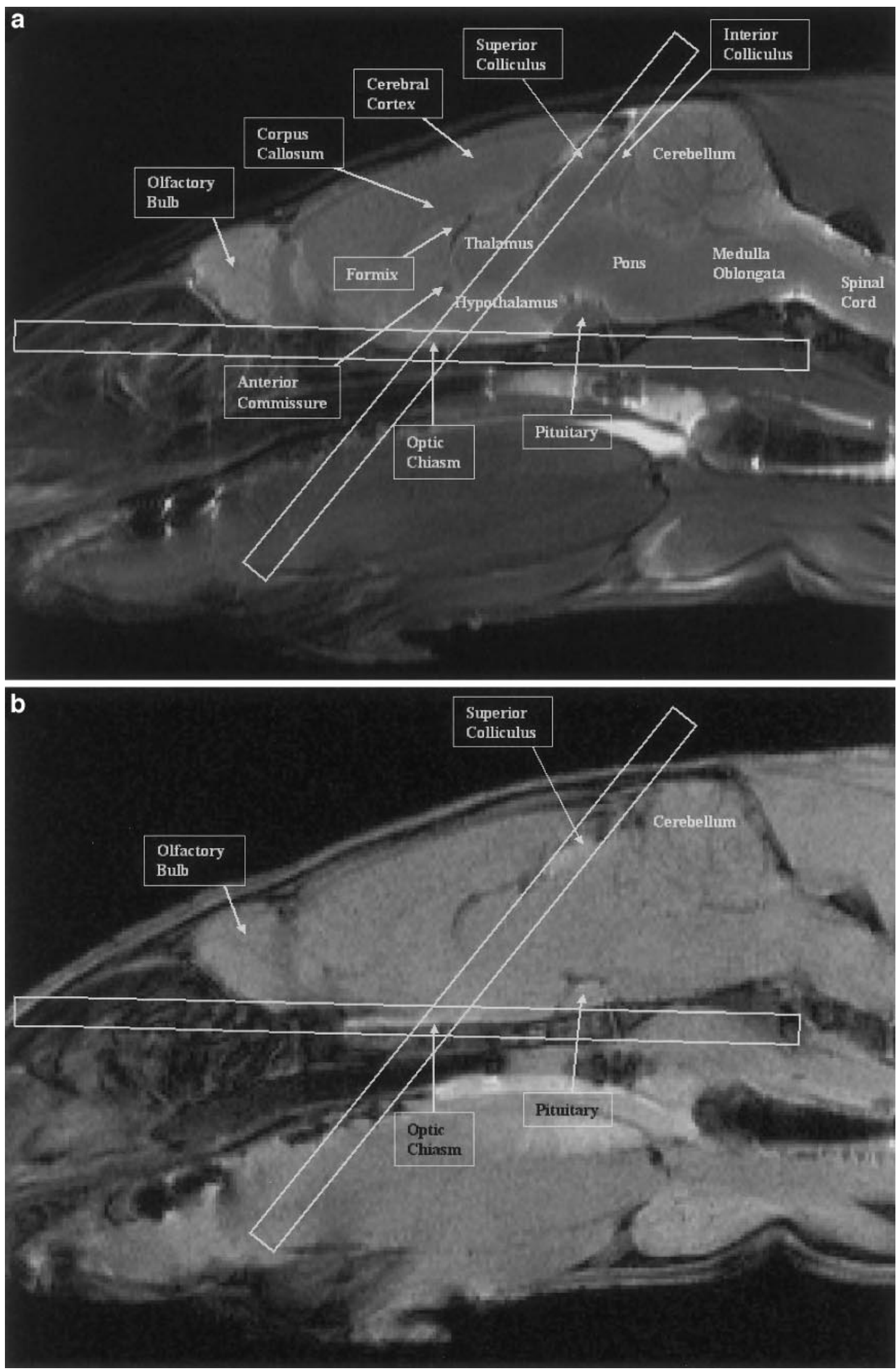

FIG. 1. Anatomical images of a rat's brain in midsagittal view. Anatomical structures are clearly shown on T2-weighted images (a). Optic chiasm and superior colliculus are identified from the enhanced regions on TIWI (b). Based on these anatomical images, orientations of two oblique slices were determined to contain the optic tracts from retina to LGN (slices are indicated with two rectangles in the Figures). The first slice covered the optic nerves from retina to optic chiasm. The second slice covered the tract from optic chiasm to bilateral LGN. 
RF
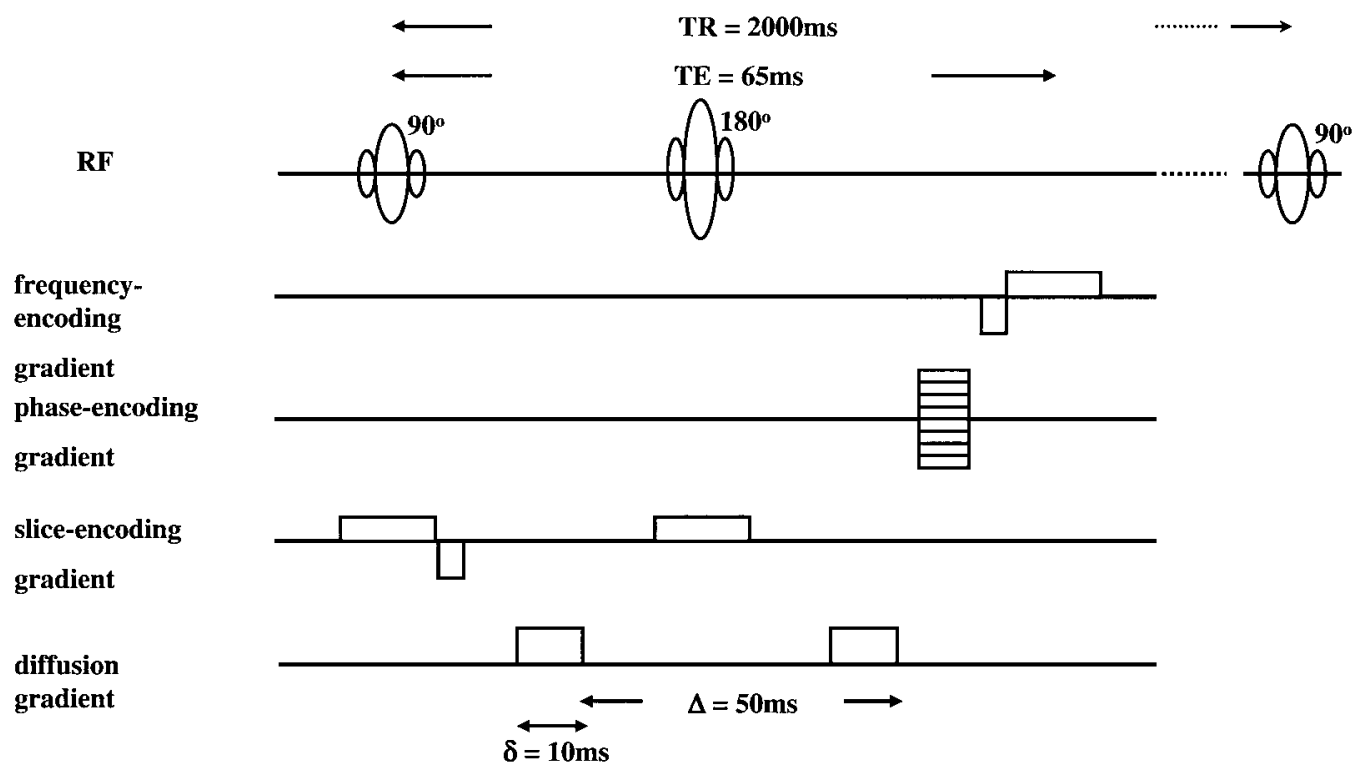

FIG. 2. Diagram of spin-echo diffusion sequence.

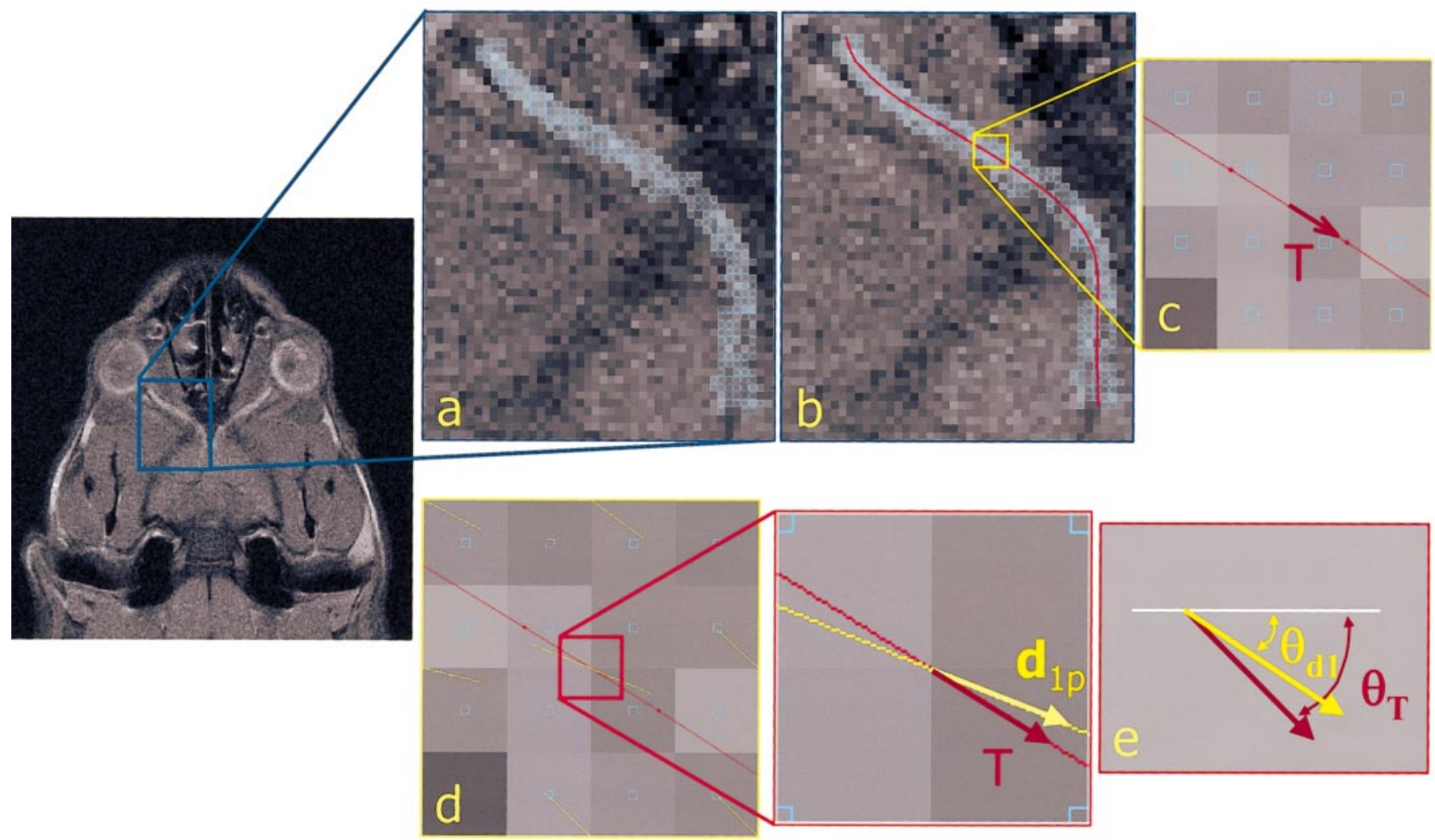

FIG. 3. Procedures of determining vectors tangential to $\mathrm{Mn}^{2+}$-enhanced tracts and computation of deviation angles. We started with an image of $\mathrm{Mn}^{2+}$-enhanced MRI with bright optic nerves as shown in the left panel. Using an appropriate magnitude threshold, the enhanced tracts were isolated (a). Sixth order least-square polynomials were fit to the enhanced pixels (b). The tangential vector $\mathrm{T}$ of any point on the tract was determined by taking spatial derivatives of the polynomials (c). The deviation angle was then computed by subtracting the polar angle of the principal diffusion eigenvector $\theta_{\mathrm{d} 1}$ from the polar angle of the tangential vector $\theta_{\mathrm{T}}$ at each corresponding position ( $\mathrm{d}$, e). 
from eddy currents, coupling of imaging with diffusion-encoding gradients, choice of diffusion-encoding schemes, and image distortion related to echo-planar readout, while they can be estimated or be corrected, would nonethel ess pol lute the ei genvector field (Mattiello et al., 1997; Bastin, 1999; Calamante et al., 1999; Porter et al., 1999; Papadakis et al., 1999). Thermal noise of MRI also introduces uncertainty to the calculated diffusion coefficients and induces sort bias of the eigenvalues (Bastin et al., 1998; Basser et al., 2000; Martin et al., 1999). All of these problems lead to inaccurate estimate of the principal eigenvector of the diffusion tensor and consequently erroneous results of tractography reconstructed from it.

To date, an appropriate gold standard for assessing the accuracy of DTMRI in defining neural fiber direction is still lacking. Histology was used as a standard reference for validating DTMRI of the myocardial fibers, yet it confronts crucial technical problems (Hsu et al., 1998; Scollan et al., 1998; Holmes et al., 2000). The histological specimens are liable to destruction and distortion during tissue preparation, changing fibers from original orientations and causing geometric mismatch between the specimens and DTMRI. In the case of neural fiber tracts, complex geometry makes histological quantification of 3-D fiber orientations in the brain even more difficult to perform.

In this paper, we developed a rat model to verify DTMRI in defining neural fiber direction in vivo. We used manganese-enhanced T1-weighted images (TIWI ) as a gold standard for estimation of the DTMRI accuracy. Divalent manganese-ion $\left(\mathrm{Mn}^{2+}\right)$ is paramagnetic and acts as an excellent MRI contrast agent (Dias et al., 1983; Kang et al., 1984). It has an ionic radius similar to that of calcium-ion $\left(\mathrm{Ca}^{2+}\right)$, which can enter cells through calcium pathways such as voltage-gated calcium channels and is confined to the intracellular compartment (Drapeau et al., 1984; Verity, 1999; Aschner et al., 1999). By administrating manganese ions into rats' olfactory bulbs and retina, Pautler et al. were among the first to demonstrate clear visualization of the neural tracts on T1WI (Pautler et al., 1998). With a rat model, we obtained enhanced optic tracts from the retina up to the lateral geniculate nucleus (LGN) and compared the tract orientation at each pixel with that derived from DTMRI. Angular deviation of the principal eigenvector of the diffusion tensor and its dependence on MR noise were assessed quantitatively.

\section{MATERIALS AND METHODS}

\section{Rat Model}

Wistar rats were anesthetized by intraperitoneal injection of sodium pentobarbital at a dose of $0.05 \mathrm{mg}$ per gram body weight. Manganese Chloride solution, 0.8 $\mathrm{mol} / \mathrm{l}$ in concentration and $2 \mu \mathrm{l}$ in amount, was infused into the vitreal cavity of rats' eyes with a micropipette needle. Ten hours after the infusion, rats were placed in prone position in an acrylic semicylindrical holder with the head fixed by foam pads. The holder was then put into a mini quadrature coil for MRI scanning. Sagittal T1- and T2-weighted images were acquired as a localizer and from which two oblique slices with different orientations were determined. One slice contained optic tracts from bilateral retina to optic chiasm, the other slice from optic chiasm to bilateral LGN. As shown in Fig. 1, we used optic chiasm and bilateral junctions of the retina and optic nerves as anatomic landmarks to determine the orientation of the first slice and used the optic chiasm and bilateral superior colliculi to determine the orientation of the second slice. In this way, optic tracts from retina to L GN were contained in two single slice planes.

\section{I maging Techniques}

MRI data were acquired using 3T MRI Biospect system (Brucker, Germany). A mini quadrature coil, 12-cm inner diameter, was used for RF transmission and reception of $\mathrm{MRI}$ signals. Registered images of TIWI and DTMRI were acquired with the same FOV $(40 \mathrm{~mm})$ and slice thickness $(1.2 \mathrm{~mm})$. We used an inversion recovery gradient echo sequence to obtain TIWI. The flip angle was $75^{\circ}, \mathrm{TR} / \mathrm{TE} / \mathrm{TI}=505 / 5.1 / 320$ $\mathrm{ms}$, and matrix size $=256$, yielding in-plane resolution of $0.15 \mathrm{~mm}$. Registered images of DTMRI were acquired with a spin-echo pulsed gradient sequence, TR/ $\mathrm{TE}=2000 / 65 \mathrm{~ms}$, matrix size $=128$, yielding in-plane resolution of $0.3 \mathrm{~mm}$. Diffusion encoding entailed six gradients in cube-octahedral orientation, i.e., $\{1,1,0\}$, $\{1,-1,0\},\{1,0,1\},\{-1,0,1\},\{0,1,1\}$, and $\{0,-1,1\}$, with gradient magnitude $|\mathbf{g}|=110 \mathrm{mT} / \mathrm{m}$, duration $\delta=$ $5 \mathrm{~ms}$, and diffusion time $\Delta=50 \mathrm{~ms}$, yielding diffusion sensitivity $b=2090 \mathrm{~s} / \mathrm{mm}^{2}$ (Fig. 2). With 16 number of excitations (nex) for each data acquisition, two slices of microscopic DTMRI were obtained in about $16 \mathrm{~h}$.

\section{Diffusion Tensor Reconstruction}

Diffusion tensor MRI reconstructs a symmetric diffusion tensor at each image pixel. The measured signal is related to the diffusion tensor $\mathbf{D}$ by

$$
\ln \left(I_{i} / I_{0}\right)=-\int_{0}^{\Delta} \mathbf{k}_{i}^{\top}(t) D k_{i}(t) d t
$$

where $I_{i}$ and $I_{0}$ represent attenuated and nonattenuated images, respectively, $\Delta$ is the diffusion time, $\mathbf{k}_{\mathrm{i}}(\mathrm{t})=(2 \pi)^{-1} \int_{\mathrm{o}}^{\Delta} \gamma \mathbf{g}_{\mathrm{i}}(\tau) \mathrm{d} \tau$, is the spatial modulation of magnetization produced by diffusion-sensitizing gradi- 
ents $\mathbf{g}_{\mathrm{i}}$ in i-th direction, where $\mathrm{i}=1,2, \ldots, 6$, and $\gamma$ is the proton gyromagnetic ratio. The diffusion cross terms induced by the imaging gradients were about 25 $\mathrm{s} / \mathrm{mm}^{2}$, approximately $1 \%$ of the $\mathrm{b}$ value, and were neglected in diffusion tensor computation. By measuring this attenuation with spatial modulations in six directions and an image of null gradient, the six coefficients of the diffusion tensor were solved at each pixel by algebraic inversion of b matrix (Basser et al., 1998). The principal eigenvector $\mathbf{d}_{1}$, namely the eigenvector of the diffusion tensor associated with the largest eigenvalue, was then determined by diagonalizing the tensor matrix.

\section{Registration Techniques}

We registered $\mathrm{Mn}^{2+}$-enhanced $\mathrm{MRI}$ with DTMRI by acquiring them in one study session, and with the same slice orientation, and FOV. Since the matrix sizes for $\mathrm{Mn}^{2+}$-enhanced MRI and for DTMRI were $256^{2}$ and $128^{2}$, respectively, the two images were coregistered for every $2 \times 2$ pixels in $\mathrm{Mn}^{2+}$-enhanced MRI corresponding to 1 pixel in DTMRI.

\section{Deviation Angles}

To quantify the accuracy of DTMRI in defining axonal fiber orientation, we registered $\mathrm{Mn}^{2+}$-enhanced TIWI with DTMRI and computed the deviation angle between $\mathbf{d}_{1}$ projection in the image plane, $\mathbf{d}_{1 p}$, and the tangential vector of an optic tract at the same location. To obtain the tangential vector, we segmented the enhanced tracts with a magnitude threshold (Fig. 3a), fit the enhanced pixels with a 6th order least-square polynomials by the principle of least curvature of fiber tracts (Poupon et al., 2000) (Fig. 3b), and determined the tangential vector of any point on the tract by taking spatial derivate of the polynomials (Fig. 3c). To compare $\mathbf{d}_{1 p}$ with the tangential vector, $\mathbf{d}_{1 p}$ at each corresponding position of the polynomial fitting curve was computed by linear interpolation of $\mathbf{d}_{1 p}$ in the closest neighborhood pixels. The deviation angle was then computed by subtracting the polar angle of $\mathbf{d}_{1 p}$ from the polar angle of the tangential vector (Figs. 3d and 3e). The observed variance of deviation angles $\sigma^{2}$ was analyzed over all samples.

\section{Variance Analysis}

To investigate the dependence of deviation angles on MRI noise we assumed that the observed variance $\sigma^{2}$ consists of two parts:

$$
\sigma^{2}=\sigma_{\mathrm{M}}^{2}+\sigma_{\mathrm{R}}^{2}
$$

where $\sigma_{\mathrm{M}}^{2}$ is the variance due to white noise from MRI system and $\sigma_{\mathrm{R}}^{2}$ is the residual variance due to the bias other than white noise. We further assume that $\sigma_{\mathrm{M}}^{2}$ follows stochastic behavior, namely, $\sigma_{\mathrm{M}}^{2}$ is inversely proportional to the square of SNR, whereas $\sigma_{\mathrm{R}}^{2}$ is independent of noise. In this case, the variance of deviation angles with half of the original SNR, $\sigma_{(1 / 2 S N R)}^{2}$ is

$$
\sigma_{(1 / 2 \mathrm{SNR})}^{2}=\sigma_{\mathrm{M}(1 / 2 \mathrm{SNR})}^{2}+\sigma_{\mathrm{R}}^{2}=4 \sigma_{\mathrm{M}}^{2}+\sigma_{\mathrm{R}}^{2}
$$

where $\sigma_{(1 / 2 \mathrm{SNR})}^{2}$ can be measured from one fourth of the data set (4 nex) of DTMRI. Having measured $\sigma^{2}$ and $\sigma_{(1 / 2 \mathrm{SNR})}^{2}$, we determined $\sigma_{\mathrm{M}}^{2}$ and $\sigma_{\mathrm{R}}^{2}$ from $\mathrm{Eqs}$. (2) and (3).

To validate stochastic behavior of $\sigma_{\mathrm{M}}^{2}$, we added Rayleigh noise to the magnitude of diffusion-attenuated images and obtained different levels of SNR decreasing incrementally from original value of 30 down to 5 . F or each level of SNR, we measured the variance from MR noise, i.e., $\sigma_{M,(S N R=n)}^{2}$ for $n=5, \ldots, 30$, and obtained a plot of root-mean-square (rms) error $\sigma_{\mathrm{M},(\mathrm{SNR}=\mathrm{n})}$ against SNR. Theoretical values of the variance from MR noise at a given level SNR, $\sigma_{\mathrm{M}}^{2}(\mathrm{SNR})$, were also computed based on the stochastic assumption:

$$
\sigma_{\mathrm{M}}^{2}(\mathrm{SNR})=\sigma_{\mathrm{M},(\mathrm{SNR}=30)}^{2} \times(30 / \mathrm{SNR})^{2} .
$$

Values of $\sigma_{\mathrm{M}}(\mathrm{SNR})$ were then compared with the measured values $\sigma_{\mathrm{M},(\mathrm{SNR}=\mathrm{n})}$ for each corresponding level of SNR.

\section{RESULTS}

\section{$\mathrm{Mn}^{2+}$-E nhanced Optic Tracts and DTMRI}

A total of four Wistar Rats were studied and seven optic tracts were obtained for analysis. Manganeseenhanced T1WI obtained $10 \mathrm{~h}$ after injection show good enhancement of the optic tracts from bilateral retina through optic chiasm to LGN. The tract length was 22 $\mathrm{mm}$ on average, indicating the speed of transport of about $2.2 \mathrm{~mm}$ per hour. There was no enhancement along the optic tracts beyond LGN. Figure 4 shows $\mathrm{Mn}^{2+}$-enhanced T1WI superimposed with $\mathbf{d}_{1 p}$ maps. Orientations of $\mathbf{d}_{1 p}$ on the optic tracts clearly show a parallel relationship with the tracts. Such parallel relationship disappears in the optic chiasm and the LGN, probably owing to fiber bundle crossing in these regions that makes directions of $\mathbf{d}_{1 \mathrm{p}}$ ambiguous. This was evidenced by significant difference in the diffusion fractional anisotropy in the optic chiasm, 0.63 ( \pm 0.26$)$, and in the LGN, $0.36( \pm 0.18)$ as compared with that in the optic tracts, $0.84( \pm 0.24)$.

\section{Deviation Angles}

For each enhanced optic tract deviation angles between $\mathbf{d}_{1 p}$ and tangential vectors of the tracts were analyzed. Table 1 lists mean and standard deviation of 

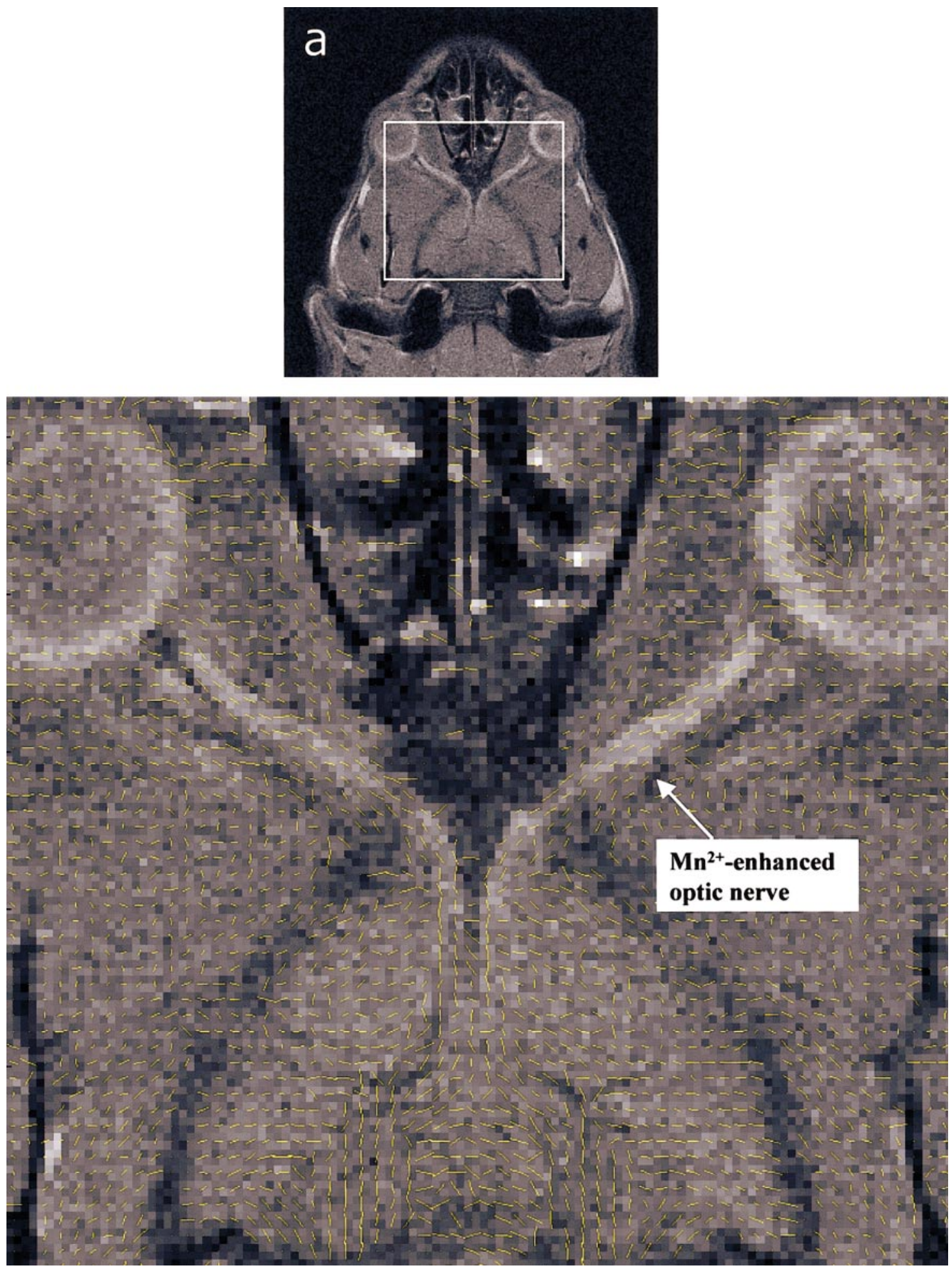

FIG. 4. I mages of $\mathrm{Mn}^{2+}$-enhanced optic tracts superimposed with principal eigenvector maps of the diffusion tensors. The magnified images are the zoom-in regions of interest enclosed by rectangles in the images on top. The superimposed images show that (a and b), at corresponding positions, the principal diffusion eigenvectors (indicated by yellow segments) are mostly parallel to the enhanced tracts. To distinguish the tract structures from the adjacent tissues, the length of each yellow segment was rescaled according to the fractional anisotropy of the diffusion tensor at that position. Having registered $\mathrm{Mn}^{2+}$-enhanced $\mathrm{MRI}$ with the images of the principal diffusion eigenvectors, deviation angles can be computed by direct comparison between tract orientations and the principal diffusion eigenvectors at each pixel as described in the legend of Fig. 3. 

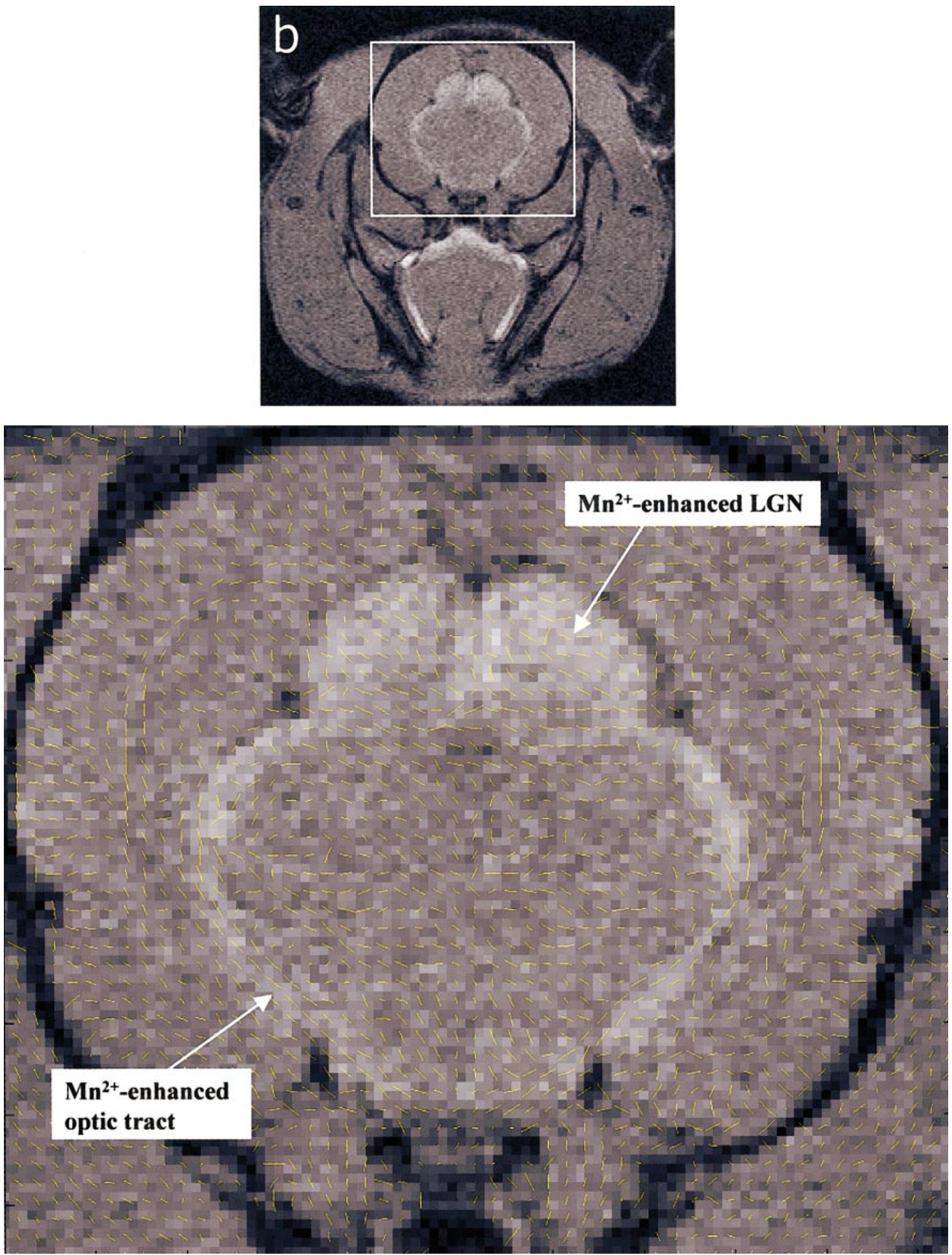

FIG. 4-Continued

deviation angles for each tract and in aggregation. The histogram of deviation angles approximates normal distribution with the mean $=-1.11^{\circ}$ and the variance $\sigma^{2}=\left(13.27^{\circ}\right)^{2}$ (Fig. 5).
Noise Estimate

A plot of the rms error from MR noise versus SNR is shown in Fig. 6. The experimental curve obtained from 
TABLE 1

Mean and Standard Deviation of Deviation Angles for Each Optic Tract and in Total

\begin{tabular}{cccc}
\hline Tract number & Number of pixels & Mean & Standard deviation \\
\hline 1 & 24 & $-6.4^{\circ}$ & $10.57^{\circ}$ \\
2 & 26 & $2.75^{\circ}$ & $12.16^{\circ}$ \\
3 & 24 & $-5.25^{\circ}$ & $15.02^{\circ}$ \\
4 & 21 & $-0.68^{\circ}$ & $16.19^{\circ}$ \\
5 & 20 & $-1.79^{\circ}$ & $15.49^{\circ}$ \\
6 & 19 & $3.11^{\circ}$ & $13.21^{\circ}$ \\
7 & 22 & $1.16^{\circ}$ & $9.38^{\circ}$ \\
Total & 156 & $-1.11^{\circ}$ & $13.27^{\circ}$ \\
\hline
\end{tabular}

the measured values of $\sigma_{\mathrm{M},(\mathrm{SNR}=\mathrm{n})}$ agrees closely with the theoretical curve of $\sigma_{\mathrm{M}}$ (SNR) from SNR of 10 to 30. The correlation coefficient $\left(r^{2}\right)$ is $0.98(P<0.001)$. When SNR is smaller than 10 , the experimental curve deviates from the theoretical curve indicating break down of the stochastic assumption.

Analysis of the variance with half of the original SNR gives $\sigma^{2}{ }_{(1 / 2 \mathrm{SNR})}=\left(22.82^{\circ}\right)^{2}$. With this and knowing that $\sigma^{2}=\left(13.27^{\circ}\right)^{2}$, we obtain $\sigma_{\mathrm{M}}^{2}=\left(10.72^{\circ}\right)^{2}$ and $\sigma_{\mathrm{R}}^{2}=$ $\left(7.82^{\circ}\right)^{2}$ by solving Eqs. (2) and (3).

\section{DISCUSSION}

By spatially registering DTMRI with images of $\mathrm{Mn}^{2+}$-enhanced optic tracts, this study presented the first validation of the principal eigenvector of the dif- fusion tensor in defining axonal fiber orientation. We found an rms deviation of $13.27^{\circ}$ between DTMRI $(\mathrm{SNR}=30)$ and $\mathrm{Mn}^{2+}$-enhanced optic tracts. F urther, we validated the stochastic behavior of variance of deviation, characterizing the dependence of the accuracy of DTMRI on noise.

\section{Validation of DTMRI Using $\mathrm{Mn}^{2+}$-Enhanced Optic Tracts}

Validation of DTMRI has been attempted histologically in myocardial fibers (Hsu et al., 1998; Scollan et al., 1998; Holmes et al., 2000). However, histological methods are prone to tissue distortion or destruction during procedures such as dissection, freezing, dehydration, fixation, microtoming, and thawing, which in turn lead to misregistration with DTMRI. Histological validation faces more stringent challenge in the brain owing to the complex 3-D geometry of axonal fiber tracts and the need for injecting tracers to identify specific fascicles. In contrast to histological methods, $\mathrm{Mn}^{2+}$-enhanced tracts can be readily acquired under identical conditions and in exact spatial registration with DTMRI. Moreover, comparison between registered diffusion and $\mathrm{Mn}^{2+}$-enhanced tracts can be performed over the whole tracts, eliminating the concern of sampling bias in histological methods that only select certain areas for comparison.

In our study, DTMRI has a diffusion length of approximately $15 \mu \mathrm{m}$, comparable to the scale of axonal fibers that is about $10 \mu \mathrm{m}$ in diameter. On the other

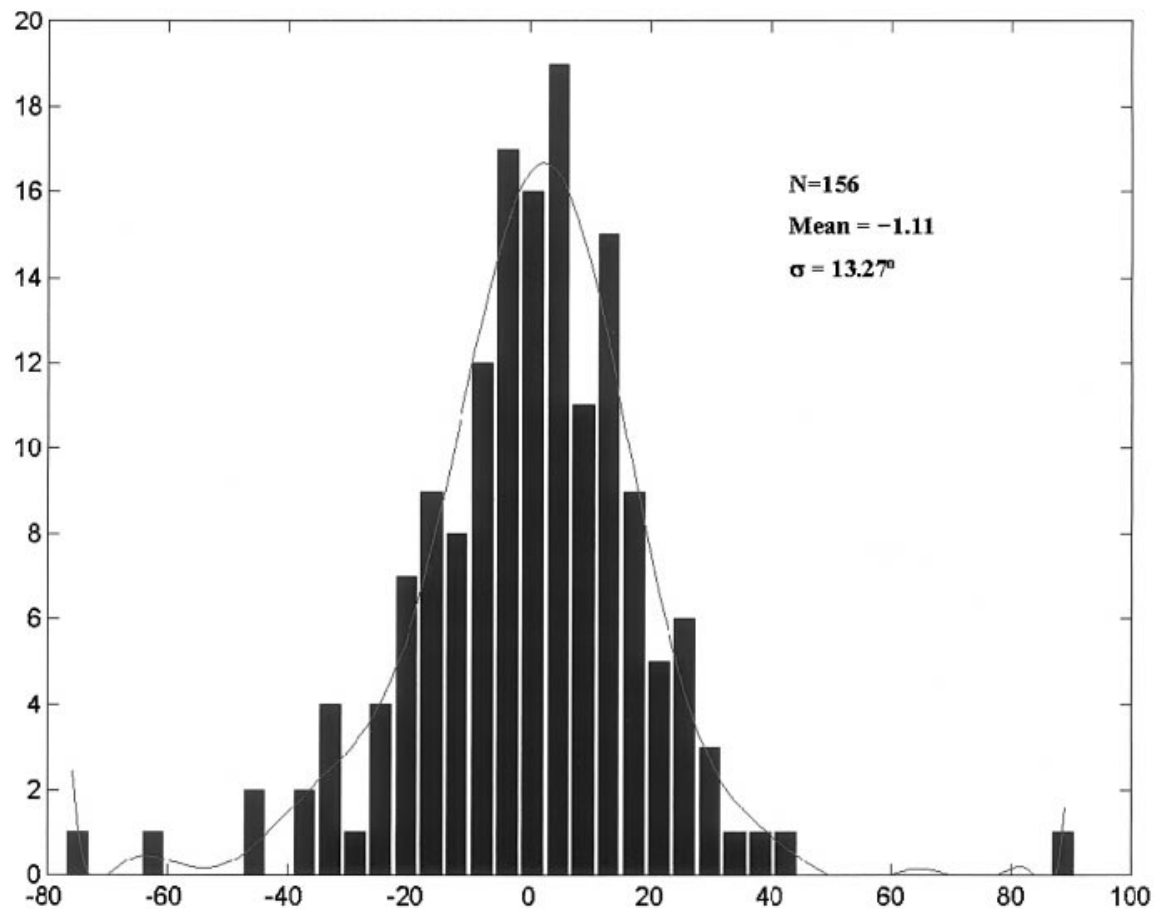

FIG. 5. Histogram of deviation angles approximates normal distribution with the mean $=-1.11^{\circ}$ and the variance $\sigma^{2}=\left(13.27^{\circ}\right)^{2}$. 


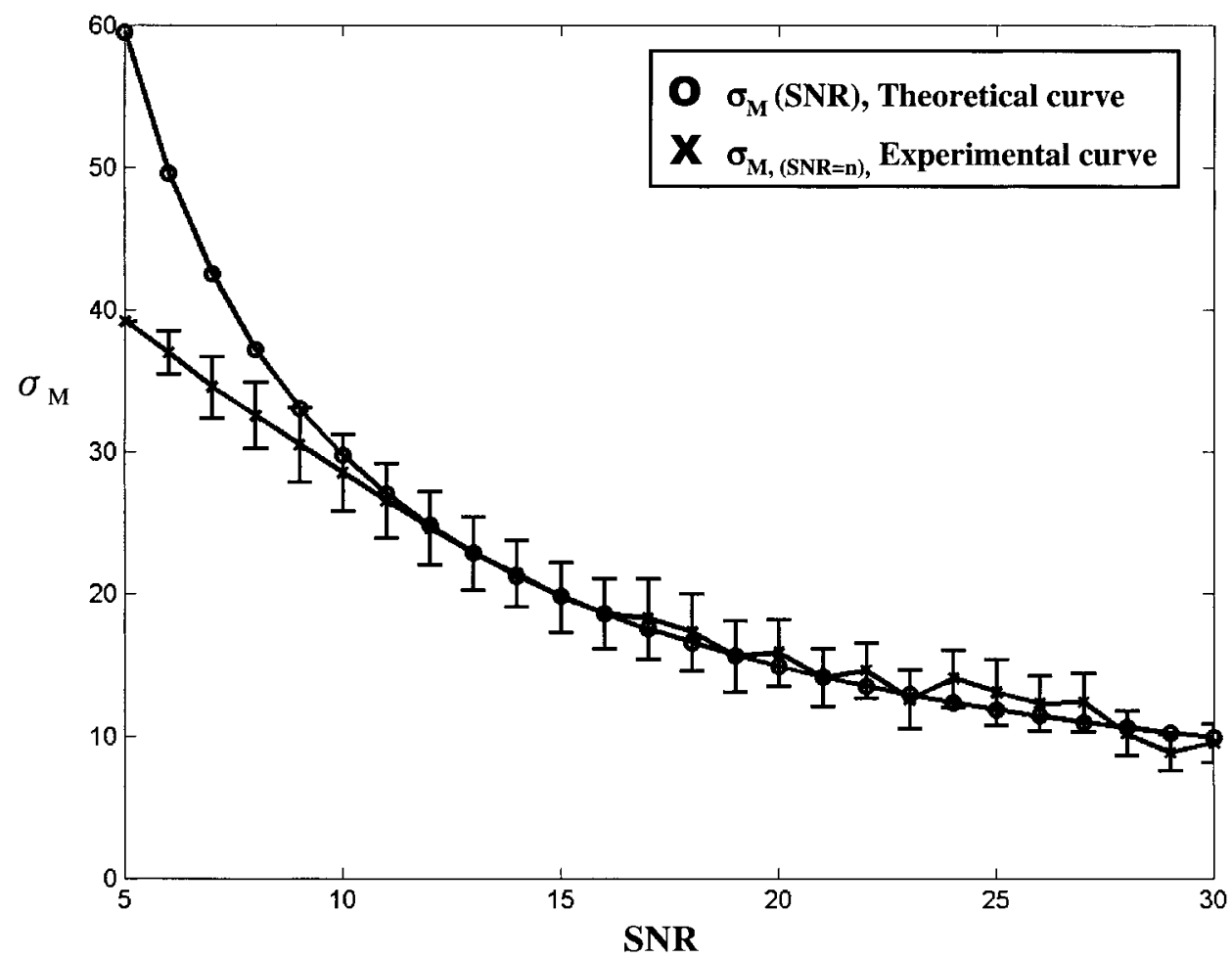

FIG. 6. A plot of rms angular errors from MR noise $\sigma_{\mathrm{M}}$ with respect to SNR: comparison between theoretical and experimental values. The error bar for each experimental value is the standard deviation of $\sigma_{\mathrm{M}}$ among seven optic nerves. The experimental curve matches the theoretical curve when SNR is greater than 10. The correlation coefficient $\left(r^{2}\right)$ between the experimental and the theoretical values is 0.98 $(\mathrm{P}<0.001)$. The plot indicates that under SNR of 10 or greater, the dependence of the standard deviation of deviation angles on SNR approximates stochastic behavior, as assumed.

hand, the resolution of $\mathrm{Mn}^{2+}$-enhanced images is 150 $\mu \mathrm{m}$. Such resolution is far too low to resolve individual axonal fibers. The discrepancy of resolution between two modalities would raise concern about the legitimacy of comparing DTMRI with $\mathrm{Mn}^{2+}$-enhanced tracts. However, according to the hierarchical organization of fiber anatomy, the direction of a gross fiber bundle should follow the orientation of constituent fibers. This parallel relation has been verified in the human brain by a comparison between individual fiber orientations with scanning electron microscopy and gross fiber bundle orientations with polarized light microscopy (Axer et al., 2000).

\section{Rationales of Using $\mathrm{Mn}^{2+}$-Enhanced Optic Tracts as a Reference}

The reasons for choosing rats' optic tracts as a reference for DTMRI validation are as follows. First, the length and size of the optic tracts are optimal. The length of optic tracts from retina to $L G N$ in rats is about $22 \mathrm{~mm}$. It takes about 10 hours for $\mathrm{Mn}^{2+}$ ions to cover the whole length. Acquisition of T1WI at this time still shows strong enhancement of the whole tracts; the enhancement would decay substantially if images had to be acquired $48 \mathrm{~h}$ after injection. The diameter of the optic tracts is about $1 \mathrm{~mm}$, equivalent to 6-pixel wide on TIWI and 3-pixel wide on DTMRI. This size is adequate for DTMRI to resolve the optic tracts without serious problems of partial volume effect. Second, direct access to the retina through intravitreal injection is possible; this obviates the need for stereotaxic puncture or dissection. Third, the entire optic tracts can be easily revealed in two single planes, one covering the tracts from the retina, through optic nerves to the chiasm, the other covering the section from the chiasm to the LGN. The slice thickness used in this study was $1.2 \mathrm{~mm}$, approximately equal to the diameter of optic tracts of a rat, and the image planes were adjusted to parallel the optic tracts. This guarantees that through-plane deviation is negligible and that the angular comparison can be simplified from threedimension to two-dimension.

\section{Limitations}

There exist several limitations in our method. First, the pathways of optic tracts from LGN to visual cortex cannot be studied because they were not enhanced, possibly due to little cross-synaptic transmission of $\mathrm{Mn}^{2+}$ ions in LGN. Second, $\mathrm{Mn}^{2+}$ enhancement only persists for 3 days. Because of this time limit, long fiber 

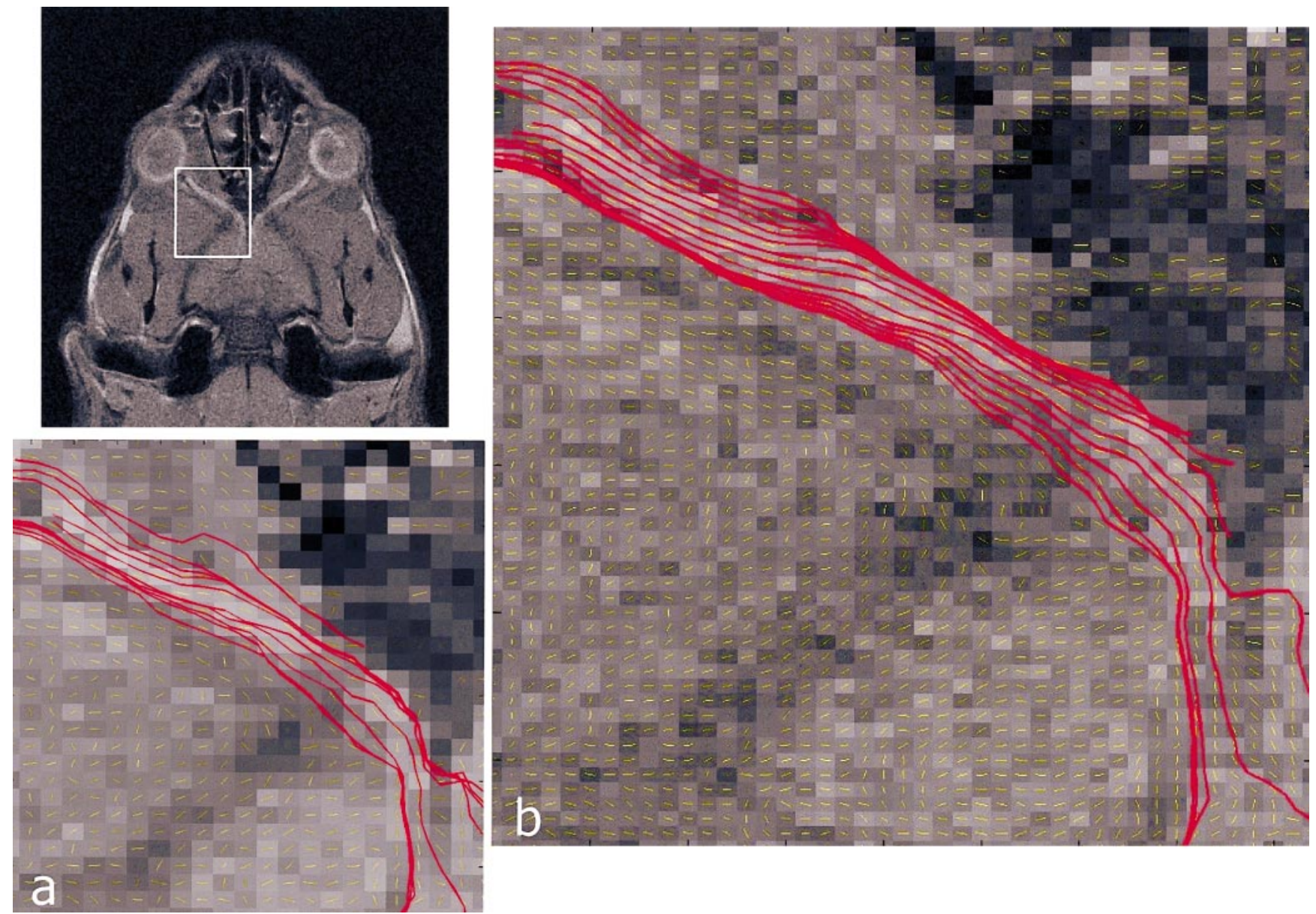

FIG. 7. The performance of diffusion tracking before and after smoothing DTMRI data set. Diffusion tractography was reconstructed from original data with $128 \times 128$ matrix size (a and c), and was compared to that reconstructed from smoothed data with $255 \times 255$ matrix size ( $b$ and d). Data smoothing was done by interpolating the magnitude data of adjacent pixels, and a commercially available reconstruction algorithm (Streamline, MATLAB 5.3) was used for diffusion tracking. To enhance fiber tracking, the length of the principal eigenvector at each pixel was scaled by the fractional anisotropy index. As compared with the original data, the diffusion tractography obtained from the smoothed data agrees more closely with the $\mathrm{Mn}^{2+}$-enhanced tracts.

tracts that require more than 3 days for $\mathrm{Mn}^{2+}$ ions to transport cannot be enhanced over the entire course. Third, the enhancement along larger fascicles is often diffuse, making tract orientations ambiguous. This was attested by our former attempt to enhance somatosensory tracts by injecting $\mathrm{Mn}^{2+}$ ions to precentral gyrus. The images showed a broad patch of faint enhancement extending from the motor cortex to thalamus.

\section{Error Estimate}

To clarify the effect of MRI noise on deviation angles, we assumed a noise reduction relation between deviation angle variance and MR noise. We found that this assumption is valid while SNR is greater than 10 . Theoretical prediction and experimental values began to show large difference when SNR is less than 10. This is probably due to relatively large perturbation from
MRI noise causing nonlinear increase in ambiguity of the principal eigenvector of the diffusion tensor (Basser et al., 2000). Based on this relationship, the rms angular error from MRI noise in our study can then be estimated; it is about $10.72^{\circ}$ under SNR of 30 . This result is consistent with previous reports in the myocardial DTMRI $\left(\sim 11^{\circ}\right)$ (Hsu et al., 1998; Tseng et al., 1999).

The residual error of our study is about $7.82^{\circ}$. This result is smaller than the error from histological method $\left(\sim 10^{\circ}\right)$ reported by Scollan (Scollan et al., 1998). While the error from histological method arises from tissue deformation and misregistration, the residual error in our study mainly comes from thresholds adopted in segmenting the $\mathrm{Mn}^{2+}$-enhanced pixels as well as artifacts related to DTMRI data acquisition methods. The results of segmentation vary with the threshold values, and there is no objective criterion for 

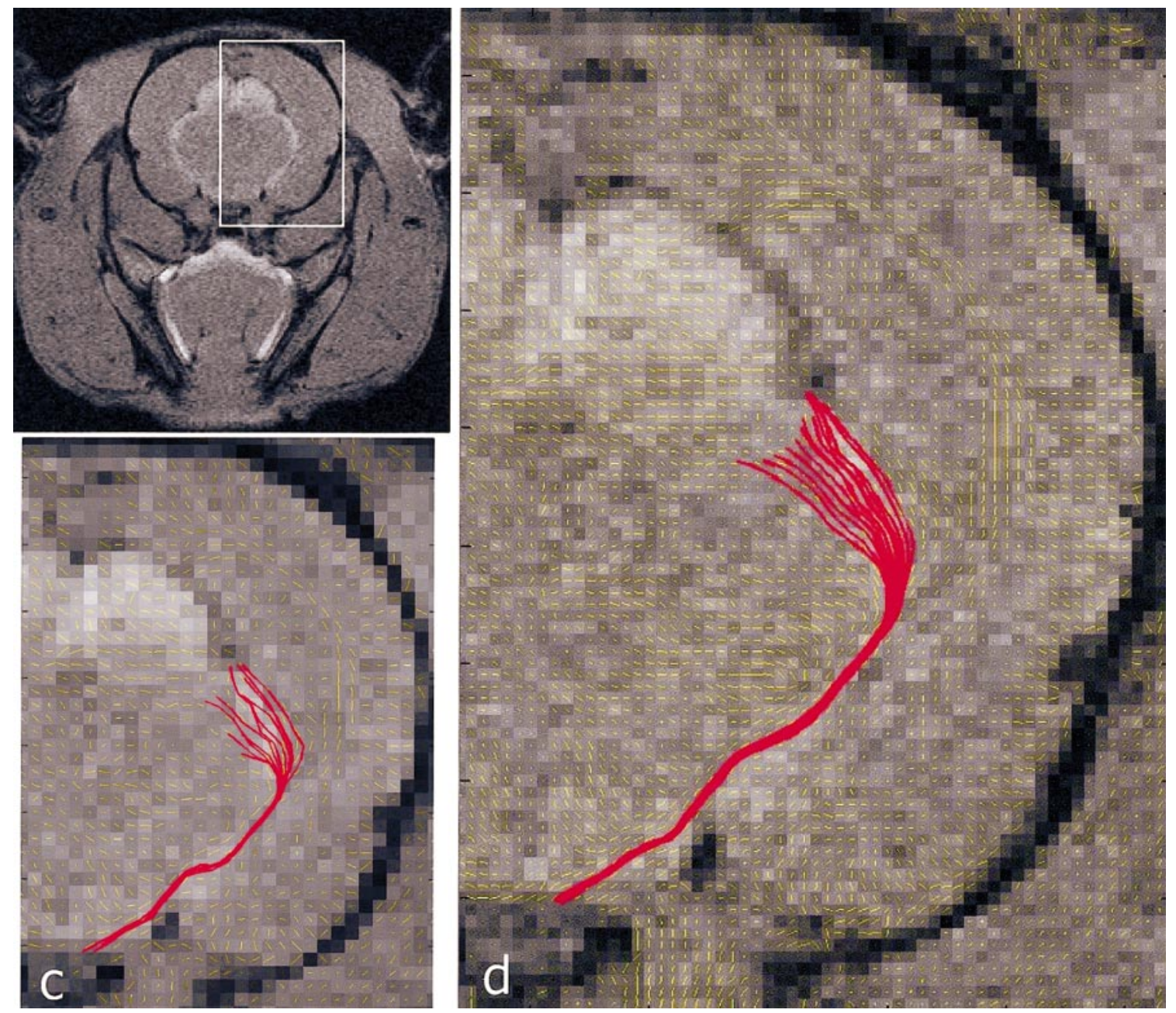

FIG. 7-Continued

determining the best thresholds. To quantify this potential bias, we used different thresholds varying from $-10 \%$ to $10 \%$ of the original threshold, and compared different segmentation results. The error from different segmentation thresholds used yields $5.11^{\circ}$ in rootmean-square sense. This error can be reduced by increasing spatial resolution of TIWI and can be readily accomplished by increasing the amount of scanning time by a few minutes only. The above estimate also shows that the residual error that is related to DTMRI data acquisition is approximately $\left(7.82^{2}-5.11^{2}\right)^{1 / 2}=$ $5.92^{\circ}$. This error, we speculate, arises from bias related to DTMRI sequence and hardware performance such as eddy current, residual diffusion cross terms, and the use of only two $b$ values $\left(b=0\right.$ and $b=2090 \mathrm{~s} / \mathrm{mm}^{2}$ ) to compute diffusivity. It follows that the total error of $13.27^{\circ}$ comprises two parts; the error related to $\mathrm{Mn}^{2+}$ enhanced T1WI constitutes $5.11^{\circ}$, and the error related to DTMRI constitutes $\left(5.92^{2}+10.72^{2}\right)^{1 / 2}=12.25^{\circ}$. The first part does not affect the accuracy of DTMRI. There- fore, our error estimate infers that the accuracy of DTMRI can be improved by increasing SNR. To have uncertainty of DTMRI within $10^{\circ}$, SNR of diffusionweighted images should be at least 40 , computed as follows:

$$
\mathrm{SNR}=\sigma_{\mathrm{M}} \times 30 \times\left(10^{2}-5.92^{2}\right)^{-1 / 2}=40 .
$$

Application of $\mathrm{Mn}^{2+}$-Enhanced Optic Tracts in Validating Diffusion Tractography

Another potential application of this technique is to validate tractography derived from DTMRI with $\mathrm{Mn}^{2+}$ enhanced tracts. There are currently different reconstruction algorithms for diffusion tractography. However, because of no gold standard, the accuracy of these algorithms can only be evaluated qualitatively by referencing to anatomy atlas. Moreover, in developing diffusion tracking, it involves data postprocessing and optimization of parameters for reconstruction algo- 
rithms. During the test procedures, it requires a gold standard to determine which approach has a better performance. As demonstrated in Fig. 7, we compared the performance of diffusion tracking using a set of DTMRI data before and after smoothing. Using $\mathrm{Mn}^{2+}$ enhanced images as a reference, it shows that data smoothing can improve the tractography results. The reason for better performance of the smoothed data may be due to noise reduction effect on smoothed pixels.

\section{CONCLUSION}

By registering images of $\mathrm{Mn}^{2+}$-enhanced optic tracts with DTMRI, we have validated the accuracy of the principal eigenvector of the diffusion tensor in defining axonal fiber orientation, and have clarified the dependence of this accuracy on SNR. Based on our error estimate, the rms error of DTMRI is less than $10^{\circ}$ if SNR is greater than 40 . With this method, the effect of data smoothing on the performance of diffusion tracking was demonstrated. Therefore, $\mathrm{Mn}^{2+}$-enhanced MRI is a feasible in vivo reference for validating DTMRI or algorithms of diffusion tractography.

\section{ACKN O WLEDG MENTS}

The authors are indebted to Dr. Chen-Tung Yen and Dr. KengChen Liang for their helpful advice to this study. This study is supported by the National Health Research Institutes Grant NHRIEX90-9018EP.

\section{REFERENCES}

Aschner, M., Vrana, K. E., and Zheng, W. 1999. Manganese uptake and distribution in the central nervous system (CNS). Neurotoxicology 20(2-3): 173-180.

Axer, M., Berks, G., and Keyserlingk, D. G. V. 2000. Visualization of nerve fiber orientation in gross histological sections of the human brain. Microsc. Res. Technique 51: 481- 492.

Basser, P., Mattiello, J ., and LeBihan, D. 1994. MR diffusion tensor spectroscopy and imaging. Biophys. J . 66: 259-267.

Basser, P., and Pierpaoli, C. 1998. A simplified method to measure the diffusion tensor from seven MR images. Magn. Reson. Med. 39: 928-934.

Basser, P., and Pajevic, S. 2000. Statistical artifacts in diffusion tensor MRI (DT-MRI) caused by background noise. Magn. Reson. Med. 44: 41-50.

Basser, P., Pajevic, S., Pierpaoli, C., Duda, J ., and Aldroubi, A. 2000. In vivo fiber tractography using DT-MRI data. Magn. Reson. Med. 44: 625- 632.

Bastin, M. E. 1999. Correction of eddy current-induced artefacts in diffusion tensor imaging using iterative cross-correlation. Magn. Reson. Imaging 17: 1011-1024.

Bastin, M. E., Armitage, P. A., and Marshall, I. 1998. A theoretical study of the effect of experimental noise on the measurement of anisotropy in diffusion imaging. Magn. Reson. Imaging 16: 773785.

Beaulieu, C., and Allen, P. S. 1994. Determinants of anisotropic water diffusion in nerves. Magn. Reson. Med. 31: 394-400.
Calamante, F., Porter, D. A., Gadian, D. G., and Connelly, A. 1999. Correction for eddy current induced Bo shifts in diffusion-weighted echo-planar imaging. Magn. Reson. Med. 41: 95-102.

Conturo, T. E., Lori, N. F., Cull, T. S., Akbudak, E., Snyder, A. Z., Shimony, J . S., McKinstry, R. C., Burton, H., and Raichle, M. E. 1999. Tracking neuronal fiber pathways in the living human brain. Proc. Natl. Acad. Sci. USA 96: 10422-10427.

Dejerine. 1895. Anatomie Des Centres Nerveux, Paris.

Dias, M. H., Gaggelli, and E. Lauterbur, P. C. 1983. Paramagnetic contrast agents in nuclear magnetic resonance medical imaging. Semin. Nud. Med. 13: 364-376.

Douek, P., Turner, R., Pekar, J ., Patronas, N., and LeBihan, D. 1991. MR color mapping of myelin fiber orientation. J . Comput. Assist. Tomogr. 15: 923-929.

Drapeau, P., and Nachshen, D. A. 1984. Manganese fluxes and manganese-dependent neurotransmitter release in presynaptic nerve endings isolated from rat brain. J . Physiol. 348: 493-510.

Friston, K. J ., and Frackowiak, R. S. J . 1997. I mages of the future: A Philosophical Coda. In Human Brain Function (R. S. J . Frackowiak, K. J. Friston, C. D. Frith, R. J. Dolan, and J . C. Mazziotta, Eds.), pp. 487-517. Academic Press, San Diego.

Holmes, A. A., Scollan, D. F., and Winslow, R. L. 2000. Direct histological validation of diffusion tensor $\mathrm{MRI}$ in formaldehyde-fixed myocardium. Magn. Reson. Med. 44: 157-161.

Hsu, E. W., Muzikant, A. L., Matulevicius, S. A., Penland, R. C., and Henriquez C. S. 1998. Magnetic resonance myocardial fiber-orientation mapping with direct histological correlation. Am. J . Physiol. 43: H1627-H 1634.

J ones, D. K., Simmons, A., Williams, S. C. R., and Horsfield, M. A. 1999. Non-invasive assessment of axonal fiber connectivity in the human brain via diffusion tensor MRI. Magn. Reson. Med. 42: 37- 41.

Kang, Y. S., and Gore, J . C. 1984. Studies of tissue NMR relaxation enhancement by manganese. Dose and time dependences. I nvest. Radiol. 19(5): 399-407.

Makris, N., Worth, A. J ., Sorensen, A. G., Papadimitrious, G. M., Wu, O., Reese, T. G., Wedeen, V. J ., Davis, T. L., Stakes, J . W., Caviness, V. S., et al. 1997. Morphometry of in vivo human white matter association pathways with diffusion-weighted magnetic resonance imaging. Ann. Neurol. 42(6): 951-962.

Martin, K. M., Papadakis, N. G., Huang, C. L., Hall, L. D., and Carpenter, T. A. 1999. The reduction of the sorting bias in the eigenvalues of the diffusion tensor. Magn. Reson. I maging 17(6): 893-901.

Mattiello, J., Basser, P. J ., and LeBihan, D. 1997. The b matrix in diffusion tensor echo-planar imaging. Magn. Reson. Med. 37: 292300.

Mori, S., Crain, B., Chacko, V. P., and van Zijl, P. C. M. 1999. Three dimensional tracking of axonal projections in the brain by magnetic resonance imaging. Ann. Neurol. 45: 265-269.

Papadakis, N. G., Xing, D., Huang, C. L.-H., Hall, L. D., and Carpenter, T. A. 1999. A comparative study of acquisition schemes for diffusion tensor I maging Using MRI. J . Magn. Reson. 137: 67- 82.

Pautler, R. G., Silva, A. C., and Koretsky, A. P. 1998. In vivo neuronal tract tracing using Manganese-enhanced magnetic resonance imaging. Magn. Reson. Med. 40: 740-748.

Pierpaoli, C., and Basser, P. J . 1996. Toward a quantitative assessment of diffusion anisotropy. Magn. Reson. Med. 36: 893-906.

Porter, D. A., Calamante, F., Gadian, D. G., and Connelly, A. 1999. The effect of residual Nyquist ghost in quantitative echo-planar diffusion imaging. Magn. Reson. Med. 42: 385-392.

Poupon, C., Clark, C. A., Frouin, V., Regis, J ., Bloch, I., Le Bihan, D., and Mangin, J. 2000. Regularization of diffusion-based direction 
maps for the tracking of brain white matter fascicles. Neuroimage 12(2): 184-195.

Rye, D. B. 1999. Tracking neural pathways with MRI. Trends Neurosci. 22(9): 373-374.

Scollan, D. F., Holmes, A., Winslow, R., and Forder, J . 1998. Histological validation of myocardial microstructure obtained from diffusion tensor magnetic resonance imaging. Am. J . Physiol. 275: $\mathrm{H} 2308-\mathrm{H} 2318$.

Tseng, W. Y. I., Reese, T. G., Smith, R.N., Halprin, E., and Wedeen, V. J. 1999. Histological correlation of myocardial diffusion tensor MRI: Fibers and sheets both contribute. In VIIIth ISMRM, p. 26. Philadel phia, PA.

Tuch, D. S., Weisskoff, R. M., Belliveau, J. W., and Wedeen, V. J . 1999. High angular resolution diffusion imaging of the human brain. In VIIIth ISMRM, p. 321. Philadelphia, PA.

Turner, B., Mishkin, M., and Knapp, M. 1980. Organization of the amygdalopetal projections from modality-specific cortical association areas in the monkey. Neurology 191: 515-543.
Verity, M. A. 1999. Manganese neurotoxicity: A mechanistic hypothesis. Neurotoxicology 20(2-3): 489- 497.

Werring, D. J ., Clark, C. A., Barker, G. J ., Miller, D. H., Parker, G. J. W., Brammer, M. J ., Bullmore, E. T., Biampietro, V. P., Thompson, A. J. 1998. The structure and functional mechanisms of motor recovery: Complementary use of diffusion tensor and functional magnetic resonance imaging in a complementary use of the internal capsule. J . Neurol. Neurosurg. Psychiatry 65: 863- 869.

Wiegell, M. R., Larsson, H. B. W., and Wedeen, V. J . 2000. Fiber crossing in human brain depicted with diffusion tensor MR imaging. Radiology 217(3): 897-903.

Young, M., Scannell, J ., and Burns, G. 1995. TheAnalysis of Cortical Connectivity. Neuroscience Intelligence Unit, Springer-Verlag.

Xue, R., van Zijl, C. M., Crain, B. J ., Solaiyappan, M., and Mori, S. 1999. In vivo three-dimensional reconstruction of rat brain axonal projections by diffusion tensor imaging. Magn. Reson. Med. 42: 1123-1127. 